\title{
Desarrollo sostenible desde la acción por el clima: interrelación de los ODS en Chile y Uruguay
}

\author{
Sustainable Development from Climate Action: \\ interrelation of the SDGs in Chile and Uruguay
}

\section{Andrea Peroni Fiscarelli, I Daniela Miranda Contreras, II Catalina Castillo IbarraIII}

I Estudió Historia y Sociología, se doctoró en Estudios Americanos, con especialidad en Estudios Sociales y Políticos. Académica de la Universidad de Chile (UCh), en el Departamento de Sociología. Coordina el Núcleo Interdisciplinario de Investigación Evaluativa orientado a la Decisión Pública (UCh). Es consultora nacional e internacional en temas de políticas sociales, planificación, monitoreo y evaluación. $\varangle$ •aperoni@uchile.cl https://orcid.org/0000-0003-0584-7705

II Licenciada en Sociología (Universidad de Chile). Integra el Centro de Investigación para la Gestión Integrada del Riesgo de Desastres (CIGIDEN) en la línea de cultura del desastre y gobernanza del riesgo. Consultora nacional e internacional de la FAO en temas de monitoreo y evaluación, cambio climático, género y gestión del conocimiento. $\varangle \cdot$ danimirandacontreras@gmail.com https://orcid.org/0000-0001-8829-7539

III Licenciada en Sociología (Universidad de Chile). Integra el Centro de Ciencia del Clima y la Resiliencia (CR)2 en la línea de gobernanza e interfaz ciencia-política. Tiene intereses y ejerce docencia en áreas de políticas públicas, evaluacion y cambio climático, especialmente en materia de adaptación. Cuenta con experiencia en temáticas de género y medición estadística. $\varangle \cdot$ catacastilloib@gmail.com https://orcid.org/0000-0002-8371-2702

RECIBIDO: 26.8 .2020

ACEPTADO: 8.11 .2020

\section{Resumen}

Este artículo aborda la interacción de los ODS, según las políticas y planes gubernamentales identificados en los informes nacionales voluntarios y los Objetivos de Desarrollo Sostenible (ODS), de Chile y Uruguay (2019), en especial, en referencia al ODS 13, «adoptar medidas urgentes para combatir el cambio climático y sus efectos». El análisis da 
cuenta de las posibilidades de vinculación entre objetivos de manera interrelacionada con los otros ODS, a fin de evaluar la viabilidad de un modelo de desarrollo integral y sostenible.

Palabras clave: cambio climático, desarrollo sostenible, Chile, Uruguay.

\section{Abstract}

This paper examines the interaction between public policies and plans and the Sustainable Development Goals (SDGs). For this purpose, this paper analyzes the instruments indicated in the Voluntary National Reports of Chile and Uruguay (2019), especially those referred in ODS 13: Take urgent action to combat climate change and its impacts. The analysis carried out shows the possibilities of fulfilment that are presented from SDG 13, in an interrelated way with the other SDGs, in order to evaluate the viability of an integral and sustainable development model.

Keywords: climate change, sustainable development, Chile, Uruguay.

\section{Introducción}

El desarrollo sostenible refiere al tipo de desarrollo que satisface las necesidades del presente sin comprometer la capacidad de las futuras generaciones y garantiza el equilibrio entre el crecimiento económico, el cuidado del medio ambiente y el bienestar social. La ausencia de tal tipo de desarrollo pone en evidencia las consecuencias medioambientales negativas del desarrollo económico y la globalización (Comisión Mundial sobre el Medio Ambiente y el Desarrollo, 1987), así como sus repercusiones en el desarrollo social.

La mirada integral sobre el desarrollo se encuentra presente en los ODS, «una agenda universal, aplicable a todos los países y a todas las personas, con un reconocimiento explícito de que la acción colectiva internacional es necesaria en muchas cuestiones» (Programa de las Naciones Unidas para el Desarrollo [PNUD], 2017, p. 4). Dicha agenda aborda todos los ámbitos del desarrollo, expresados en sus 17 objetivos.

Los Objetivos de Desarrollo Sostenible (ODS) son:

1. Erradicar la pobreza en todas sus formas en todo el mundo.

2. Poner fin al hambre, conseguir la seguridad alimentaria y una mejor nutrición, y promover la agricultura sostenible.

3. Garantizar una vida saludable y promover el bienestar para todos y todas en todas las edades.

4. Garantizar una educación de calidad inclusiva y equitativa, y promover las oportunidades de aprendizaje permanente para todos. 
5. Alcanzar la igualdad entre los géneros y empoderar a todas las mujeres y niñas.

6. Garantizar la disponibilidad y la gestión sostenible del agua y el saneamiento para todos.

7. Asegurar el acceso a energías asequibles, fiables, sostenibles y modernas para todos.

8. Fomentar el crecimiento económico sostenido, inclusivo y sostenible, el empleo pleno y productivo, y el trabajo decente para todos.

9. Desarrollar infraestructuras resilientes, promover la industrialización inclusiva y sostenible, y fomentar la innovación.

10. Reducir las desigualdades entre países y dentro de ellos.

11. Conseguir que las ciudades y los asentamientos humanos sean inclusivos, seguros, resilientes y sostenibles.

12. Garantizar las pautas de consumo y de producción sostenibles.

13. Tomar medidas urgentes para combatir el cambio climático y sus efectos.

14. Conservar y utilizar de forma sostenible los océanos, mares y recursos marinos para lograr el desarrollo sostenible.

15. Proteger, restaurar y promover la utilización sostenible de los ecosistemas terrestres, gestionar de manera sostenible los bosques, combatir la desertificación y detener y revertir la degradación de la tierra, y frenar la pérdida de diversidad biológica.

16. Promover sociedades pacíficas e inclusivas para el desarrollo sostenible, facilitar acceso a la justicia para todos y crear instituciones eficaces, responsables e inclusivas a todos los niveles.

17. Fortalecer los medios de ejecución y reavivar la alianza mundial para el desarrollo sostenible.

En el presente texto se profundiza en el ODS 13, asociado al cambio climático, porque «el cambio global y el cambio climático son realidades instaladas definitivamente entre nosotros, no ya como problemas del futuro, sino como una realidad a la cual nos hemos de adaptar y un desafío al que hemos de responder» (Duarte, 2006, p. 22). Por por consiguiente, refiere a un ámbito del desarrollo que repercute per se en los otros Objetivos.

El cambio climático es uno de los mayores desafíos de la humanidad, puesto que compromete tanto «a las personas que consciente o inconscientemente incidimos o atenuamos el problema con nuestras opciones personales y estilo de vida, como a, y de forma particularmente aguda, las generaciones futuras, nuestros hijos, nietos y sus descendientes» (Duarte, 2006, p. 23). En la actualidad no hay país que no haya experimentado los dramáticos efectos del cambio climático. 
Las emisiones de gases de efecto invernadero continúan aumentando y hoy son un $50 \%$ superiores al nivel de 1990. Las pérdidas anuales promedio causadas solo por catástrofes relacionadas al clima alcanzan a cientos de miles de millones de dólares, sin mencionar el impacto humano de las catástrofes geofísicas, el 91\% son relacionadas al clima, y que entre 1998 y 2017 tomaron la vida de 1,3 millones de personas, y dejaron a 4.400 millones heridas (PNUD, 2020)

Los ODS se dividen en tres pilares que permiten orientar el desarrollo sostenible: económico, ${ }^{1}$ social $^{2}$ y de medio ambiente. ${ }^{3}$ Este esquema, en el cual la biosfera es representada por los ODS de medio ambiente, es la base para los sistemas que soportan la vida en la Tierra y brinda tanto los servicios y recursos de los cuales dependemos. Así también, la diversidad biológica brinda «un componente central del sistema de creencias e identidades culturales en todo el mundo. Existe una fuerte relación entre la diversidad biológica y resiliencia de un ecosistema, entendiendo por tal la capacidad de afrontar el cambio y continuar desarrollándose» (Stockholm Resilience Centre, 2016, p. 6). Así, el foco principal del desarrollo sostenible es la preservación de la biosfera y sus sistemas naturales; de lo contrario, la sociedad y la economía no son viables (Lur Consultores, 2019). Como representación gráfica de esta relación, el Centro de Resiliencia de Estocolmo elaboró el siguiente esquema de la figura 1.

Dado que el cambio climático representa una amenaza para los sistemas naturales, la acción por el clima del ODS 13 se transforma en primordial para continuar con la vida de los sistemas. Se trata, entonces, de un área que presenta déficits alarmantes, consecuente de acciones previas del modelo de civilización, pero que además compromete a la Tierra hoy, y a su futuro, al bienestar de sus poblaciones. Por este carácter dinámico de acción sincrónica y asincrónica, este estudio evaluativo se ha enfocado en el análisis del ODS 13 en relación con los otros ODS.

1 Pilar económico, compuesto por los ODS 8, 9, 10 y 12.

2 Pilar social, compuesto por los ODS 1, 2, 3, 4, 5, 7, 11 y 16.

3 Pilar medio ambiente, compuesto por los ODS 6, 13, 14 y 15. 
Figura 1. Visión de la interrelación de los aspectos económicos, sociales y ambientales de los ODS

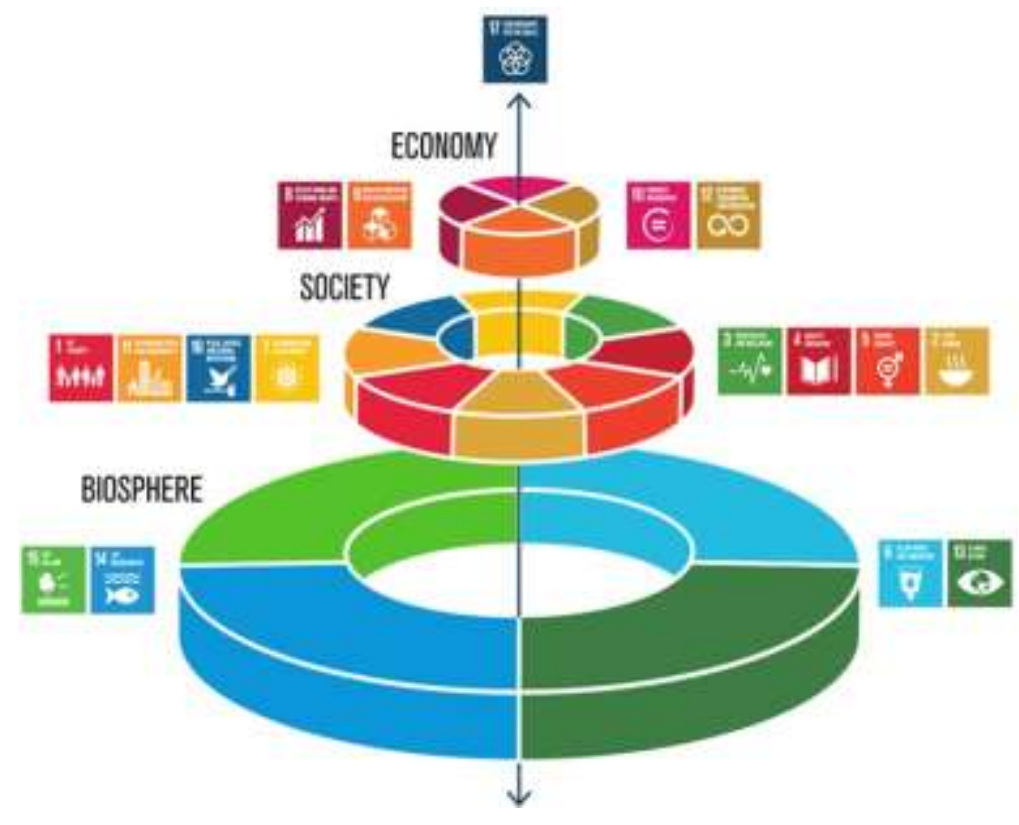

Fuente: Rockström y Sukhdev (2016).

Los ODS refieren a un modelo de desarrollo sostenible, entre cuyos principios orientadores se encuentran: i) universalidad: implica que los objetivos y las metas son relevantes para todos los gobiernos y los actores; ii) que nadie quede atrás: ningún objetivo será logrado a menos que se cumpla para todas las personas; ${ }^{4}$ iii) integración de políticas públicas: equilibrar las dimensiones de desarrollo social, crecimiento económico y protección ambiental. Un enfoque integrado implica la gestión de compensaciones (trade-offs) y la maximización de sinergias entre Objetivos (PNUD, 2017, p. 4). Si bien los tres principios son relevantes, el principio de integración entrega una clara orientación hacia la interrelación entre los mismos ODS y con los objetivos de desarrollo nacionales. Al respecto, Weitz y otros autores (2018) explican la necesidad de adoptar un marco interrelacionado para planificar la implementación de los Objetivos. «En particular, se resalta el uso para los responsables de la formulación de políticas, ya que estos pueden aumentar su comprensión de los vínculos entre los ODS y sus metas» (Lobos

El progreso debe darse independientemente del nivel de ingreso o de la presencia de exclusiones; los ODS deben beneficiar a todos. Este desafío promueve el uso de datos desagregados para comprender los desafíos sociales de cada ciudadano. 
Alva et al., 2019, p. 4). Así, la adopción de una mirada integrada permite comprender la Agenda dentro de un sistema considerando la forma en que interactúan todas las metas, y tener en cuenta:

[...] la forma en que las interacciones, entre las metas, se materializan en un contexto dado con su geografía específica, sus arreglos de gobernanza y sus opciones tecnológicas, y ofrece más oportunidades para describir y analizar cómo interactúan las metas ODS entre sí y con el sistema ODS en su conjunto y, por tanto, cómo mejor identificar acciones sinérgicas para su implementación. (Lobos et al., 2019, p. 4)

Luego de cinco años de implementación de la Agenda corresponde evaluar el nivel de cumplimiento del principio de integración. Esta evaluación permitirá observar la interrelación entre los ODS, a fin de indicar cuán interconectados se encuentran las acciones de los gobiernos con las áreas de desarrollo propuestas por los ODS, con base en el ODS 13.

La metodología desarrollada se basa en la información presentada en los informes nacionales voluntarios (INV) (Presidencia República Oriental del Uruguay, 2019; Gobierno de Chile, 2019). Los INV son elaborados por los Estados; son mecanismos de seguimiento y revisión de la Agenda 2030 a nivel nacional y subnacional. Estas revisiones nacionales son una parte esencial de las revisiones regulares que se realizan durante el Foro Político de Alto Nivel sobre el Desarrollo Sostenible (FPAN/HLPF). El objetivo de los INV es facilitar el intercambio de experiencias entre pares, incluidos los éxitos, los desafíos y las lecciones aprendidas, con miras a acelerar la implementación de la Agenda 2030. Los INV también buscan fortalecer las políticas e instituciones de los gobiernos y movilizar el apoyo de múltiples partes interesadas y fomentar alianzas para la implementación de los ODS. Específicamente, la presente investigación ha trabajado, en primer lugar, en la revisión de los correspondientes INV 2019 de Chile y Uruguay, profundizando en planes y políticas correspondientes al ODS 13. La selección de ambos países radicó en la similitud en niveles de desarrollo, en tanto, en 2013, Chile y Uruguay fueron los primeros países en América Latina clasificados por el Banco Mundial como países de altos ingresos, puesto que superaron la barrera de los USD 12.616 de PIB per capita (Banco Mundial, 2013). En segundo lugar, se analizó la información que presentan los documentos oficiales mencionados en el cumplimiento de dicho Objetivo, que corresponden a instrumentos de planificación estatal como políticas nacionales o sectoriales, planes estratégicos, planes de adaptación, lineamientos. De esta manera, el corpus documental se constituyó así: 
Figura 2. Corpus de documentos analizados

\begin{tabular}{|c|c|c|c|}
\hline \multicolumn{2}{|c|}{$\begin{array}{l}\text { Gobierno de Uruguay. } 2019 \\
\text { Informe Nacional Voluntario }\end{array}$} & \multicolumn{2}{|c|}{$\begin{array}{l}\text { Gobierno de Chile. } 2019 \\
\text { Informe Nacional Voluntario }\end{array}$} \\
\hline $\begin{array}{l}\text { Instrumentos } \\
\text { analizados }\end{array}$ & Entidad encargada & $\begin{array}{l}\text { Instrumentos } \\
\text { analizados }\end{array}$ & Entidad encargada \\
\hline $\begin{array}{l}\text { Política Energé- } \\
\text { tica Nacional } \\
(2008)\end{array}$ & $\begin{array}{l}\text { Ministerio } \\
\text { de Industria, Energía } \\
\text { y Minería. }\end{array}$ & $\begin{array}{l}\text { Política Nacional } \\
\text { para Gestión de } \\
\text { Riesgo de Desastres } \\
\text { (PNGRD) y Plan Es- } \\
\text { tratégico Nacional } \\
\text { para la Gestión de } \\
\text { Riesgo de Desastres } \\
\text { 2020-2030 } \\
\text { (PENGRD) (2020) }\end{array}$ & $\begin{array}{l}\text { Oficina Nacional de } \\
\text { Emergencia del } \\
\text { Ministerio del Inte- } \\
\text { rior y Seguridad } \\
\text { Pública (ONEMI) }\end{array}$ \\
\hline $\begin{array}{l}\text { Política Nacio- } \\
\text { nal de Aguas } \\
(2009)\end{array}$ & $\begin{array}{l}\text { Ministerio de } \\
\text { Vivienda, Ordena- } \\
\text { miento Territorial } \\
\text { y Medio Ambiente } \\
\text { (MVOTMA) }\end{array}$ & $\begin{array}{l}\text { Plan de Acción Na- } \\
\text { cional de Cambio } \\
\text { Climático 2017- } \\
2022 \text { (2017) }\end{array}$ & $\begin{array}{l}\text { División de Cambio } \\
\text { Climático del Minis- } \\
\text { terio del Medio Am- } \\
\text { biente }\end{array}$ \\
\hline $\begin{array}{l}\text { Plan Nacional } \\
\text { Ambiental para } \\
\text { el Desarrollo } \\
\text { Sostenible } \\
\text { (2018) }\end{array}$ & $\begin{array}{l}\text { Ministerio de } \\
\text { Vivienda, Ordena- } \\
\text { miento Territorial } \\
\text { y Medio Ambiente } \\
\text { (MVOTMA) y Sistema } \\
\text { Nacional Ambiental } \\
\text { (SNA). }\end{array}$ & $\begin{array}{l}\text { Plan Nacional de } \\
\text { Adaptación al Cam- } \\
\text { bio Climático (NAP) } \\
\text { (2014) }\end{array}$ & $\begin{array}{l}\text { Departamento de } \\
\text { Cambio Climático } \\
\text { del Ministerio del } \\
\text { Medio Ambiente }\end{array}$ \\
\hline $\begin{array}{l}\text { Política Nacio- } \\
\text { nal de Riesgos } \\
\text { de Desastres } \\
(2020)^{5}\end{array}$ & $\begin{array}{l}\text { Sistema Nacional de } \\
\text { Emergencias }\end{array}$ & $\begin{array}{l}\text { Plan de Adaptación } \\
\text { Sectorial Silvoagro- } \\
\text { pecuario (2013) }\end{array}$ & $\begin{array}{l}\text { Ministerios de } \\
\text { Agricultura } \\
\text { y Medio Ambiente }\end{array}$ \\
\hline $\begin{array}{l}\text { Estrategia } \\
\text { Nacional para } \\
\text { la Igualdad de } \\
\text { Género (2017) }\end{array}$ & $\begin{array}{l}\text { Ministerio de Desa- } \\
\text { rrollo Social, Insti- } \\
\text { tuto Nacional de las } \\
\text { Mujeres y Consejo } \\
\text { Nacional de Género }\end{array}$ & $\begin{array}{l}\text { Plan de Adaptación } \\
\text { para la Biodiversi- } \\
\text { dad (2014) }\end{array}$ & $\begin{array}{l}\text { Coordinado por el } \\
\text { Ministerio del Medio } \\
\text { Ambiente junto a } \\
\text { siete ministerios }\end{array}$ \\
\hline
\end{tabular}




\begin{tabular}{|c|c|c|c|}
\hline $\begin{array}{l}\text { Plan Nacional } \\
\text { de Cambio } \\
\text { Climático } \\
\text { (2017) }\end{array}$ & $\begin{array}{l}\text { Sistema Nacional } \\
\text { Ambiental (SNA), } \\
\text { Gabinete Nacional } \\
\text { Ambiental (GNA), } \\
\text { Sistema Nacional de } \\
\text { Respuesta al Cambio } \\
\text { Climático y Variabili- } \\
\text { dad (SNRCC). }\end{array}$ & $\begin{array}{l}\text { Plan de Adaptación } \\
\text { Sectorial Pesca y } \\
\text { Acuicultura (2015) }\end{array}$ & $\begin{array}{l}\text { Ministerio de Econo- } \\
\text { mía, Fomento y Tu- } \\
\text { rismo; Subsecretaría } \\
\text { de Pesca y Acuicul- } \\
\text { tura, Ministerio del } \\
\text { Medio Ambiente }\end{array}$ \\
\hline & & $\begin{array}{l}\text { Plan de Adaptación } \\
\text { Sectorial Salud } \\
\text { (2017) }\end{array}$ & $\begin{array}{l}\text { Ministerio de Salud; } \\
\text { Ministerio del Medio } \\
\text { Ambiente }\end{array}$ \\
\hline & & $\begin{array}{l}\text { Plan de Acción de } \\
\text { los Servicios de } \\
\text { Infraestructura al } \\
\text { Cambio Climático } \\
\text { (2017) }\end{array}$ & $\begin{array}{l}\text { Ministerio de Obras } \\
\text { Públicas; Ministerio } \\
\text { del Medio Ambiente }\end{array}$ \\
\hline & & $\begin{array}{l}\text { Plan de Adaptación } \\
\text { para Ciudades } \\
\text { (2018) }\end{array}$ & $\begin{array}{l}\text { Ministerio del Medio } \\
\text { Ambiente; Ministerio } \\
\text { de Vivienda y } \\
\text { Urbanismo }\end{array}$ \\
\hline
\end{tabular}

Fuente: Elaboración propia.

Se utilizó la técnica de análisis de contenido, con la que se realizó una primera identificación y descomposición de cada texto objeto de estudio en fragmentos significativos, esto es, unidades operativas, identificadas como medidas, directrices o acciones de las políticas o los planes. Posteriormente se categorizó cada unidad operativa según la mención de las áreas temáticas presentes en las metas de los otros ODS y se estableció un gradiente según tipo de vinculación. En un segundo momento se estimó el nivel de interrelación, según la frecuencia relativa, de los tipos de vinculaciones, de las unidades operativas, con relación a cada ODS, y se estableció una escala, desde la ausencia de interrelación hasta la interrelación directa, entre el ODS 13 y las metas de los otros ODS.

El proceso de análisis contó con dos escalas de valoración, a fin de identificar el nivel de vínculo entre las acciones/omisiones propuestas en los documentos y los ODS. Se generaron los gradientes que se muestran en la figura 3. 
Figura 3. Escalas niveles de interrelación entre las medidas, directrices o acciones ODS 13 y otros ODS

\begin{tabular}{|c|c|}
\hline $\begin{array}{l}\text { Primera escala, basada en las unidades } \\
\text { operativas. Se otorgó la valoración según } \\
\text { mención de área temática presente en la } \\
\text { meta de otros ODS. Manifestación explí- } \\
\text { cita del tipo de vinculación }\end{array}$ & $\begin{array}{l}\text { Segunda escala, basada en clasificación } \\
\text { de la vinculación. Se otorgó la valoración } \\
\text { por nivel de interrelación. Nivel de inter- } \\
\text { relación }\end{array}$ \\
\hline $\begin{array}{l}\text { No presenta vínculo con las me- } \\
\text { didas, directrices o acciones de } \\
\text { las políticas o planes analizados }\end{array}$ & Ausencia de interrelación \\
\hline $\begin{array}{l}\text { Se vincula de manera indirecta } \\
\text { con otras metas de los ODS. Se } \\
\text { encuentra en el ámbito de ac- } \\
\text { ción del ODS, pero no responde } \\
\text { a una meta específica. }\end{array}$ & $\begin{array}{l}\text { Interrelación tipo combinada } \\
\text { (directa e indirecta con otras } \\
\text { metas de los ODS) } \\
\text { Estimación: presencia en cada } \\
\text { ODS, de tipos de vínculos di- } \\
\text { rectos o indirectos, menor al } \\
75 \%\end{array}$ \\
\hline $\begin{array}{l}\text { Se vincula de manera directa } \\
\text { con el ODS. Contribuye directa- } \\
\text { mente al logro de una meta/a } \\
\text { del ODS relacionado }\end{array}$ & $\begin{array}{l}\text { Interrelación indirecta con } \\
\text { otras metas de los ODS } \\
\text { Estimación: presencia en cada } \\
\text { ODS, de tipos de vínculos in- } \\
\text { directos, entre el } 75 \% \text { y el } \\
100 \%\end{array}$ \\
\hline & $\begin{array}{l}\text { Interrelación directa con } \\
\text { otras metas de los ODS. } \\
\text { Estimación: presencia en cada } \\
\text { ODS, de tipos de vínculos di- } \\
\text { rectos, entre el } 75 \% \text { y el } \\
100 \% \text {. }\end{array}$ \\
\hline
\end{tabular}

Fuente: Elaboración propia. 


\section{Análisis de interrelación de los ODS respecto a los planes y políticas del ODS 13 en Chile y Uruguay}

En esta sección se presentan los resultados del análisis de contenido de las distintas políticas y programas referentes al ODS 13 de Chile y Uruguay. Se presenta el análisis para cada uno de los pilares (social, económico y de medio ambiente) y posteriormente una comparación entre estos.

\section{Análisis de Chile}

Los planes y políticas analizados corresponden a aquellos presentados como logros del ODS 13 en el Informe nacional voluntario 2019. Si bien se presentan siete planes sectoriales, se analizan solo seis, puesto que el Plan de Adaptación al Cambio Climático del Sector Energía se encuentra en fase de anteproyecto. A partir del análisis de estos instrumentos se construyó la matriz resumen de resultados (figura 4) y se elaboraron los gráficos (figura 5).

Figura 4. Chile, matriz de resultados 6

\begin{tabular}{|c|c|c|c|c|c|c|c|c|c|c|c|c|c|c|c|c|c|}
\hline Política o Plan/ODS & 1 & 2 & 3 & 4 & 5 & 6 & 7 & 8 & 9 & 10 & 11 & 12 & 13 & 14 & 15 & 16 & 17 \\
\hline $\begin{array}{l}\text { Política Nacional para Gestión de Riesgo } \\
\text { de Desastres }\end{array}$ & & & & & & & & & & & & & & & & & \\
\hline $\begin{array}{l}\text { Plan Estratégico Nacional para la Ges- } \\
\text { tión de Riesgo de Desastres }\end{array}$ & & & & & & & & & & & & & & & & & \\
\hline $\begin{array}{l}\text { Plan Nacional de Adaptación al Cambio } \\
\text { Climático (NAP) }\end{array}$ & & & & & & & & & & & & & & & & & \\
\hline $\begin{array}{l}\text { Plan de Acción Nacional de Cambio Cli- } \\
\text { mático 2017-2022 }\end{array}$ & & & & & & & & & & & & & & & & & \\
\hline $\begin{array}{l}\text { Plan de Adaptación Sectorial Silvoagro- } \\
\text { pecuario }\end{array}$ & & & & & & & & & & & & & & & & & \\
\hline Plan de Adaptación para la Biodiversidad & & & & & & & & & & & & & & & & & \\
\hline $\begin{array}{l}\text { Plan de Adaptación Sectorial Pesca y } \\
\text { Acuicultura }\end{array}$ & & & & & & & & & & & & & & & & & \\
\hline Plan de Adaptación Sectorial Salud & & & & & & & & & & & & & & & & & \\
\hline $\begin{array}{l}\text { Plan de Acción de los Servicios de Infra- } \\
\text { estructura al Cambio Climático }\end{array}$ & & & & & & & & & & & & & & & & & \\
\hline Plan de Adaptación para Ciudades & & & & & & & & & & & & & & & & & \\
\hline
\end{tabular}

Fuente: Elaboración propia. amarillo $(1,2,3,4,5,7,11,16)$; el pilar ambiental, azul $(6,13,14,15)$, el pilar económico, rojo $(8,9,10,12)$. 
Figura 5. Chile, análisis de pilares de desarrollo sostenible

Pilar económico

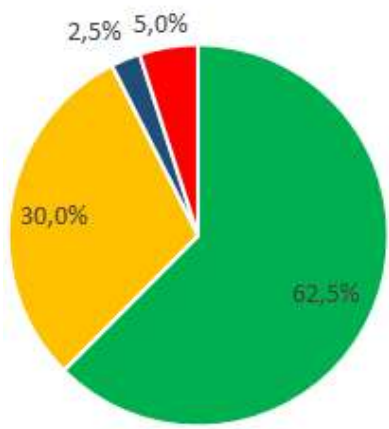

Pilar social

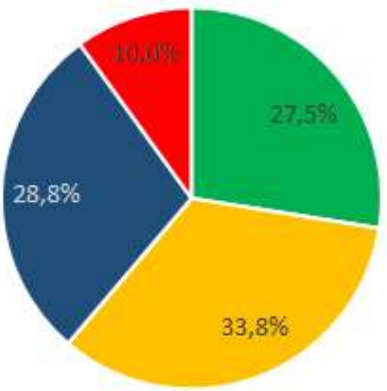

\section{Pilar medio ambiente}

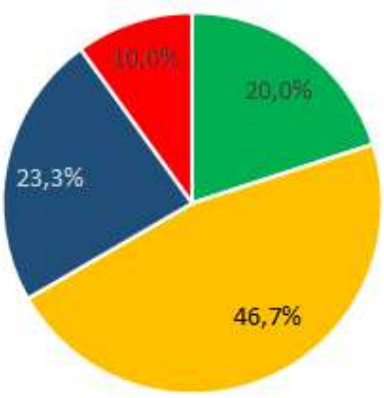

Referencias

- Interrelación directa con las metas deIODS

" Interrelación tipo combinada (directa e indirecta con las metas del ODS)

- Interrelación indirecta con las metas del ODS

- Ausencia de interrelación

Fuente: Elaboración propia.

Los gráficos de la figura 5 muestran una preponderancia de relaciones directas entre los planes y políticas del ODS 13 con las metas de los Objetivos del pilar económico $(62,5 \%)$; este pilar es el que tiene más relaciones directas con los planes y políticas del ODS 13 por un amplio margen, seguido por el pilar social con un 27,5\% y el pilar de medio ambiente con un $20 \%$ de relaciones directas. El pilar económico presenta un $30 \%$ de interrelaciones de tipo combinadas, por lo que, en combinación con las interrelaciones directas, representa un 92,5\% del total de interrelaciones entre las políticas y planes del ODS 13 con los ODS económicos.

El pilar social por su parte, manifiesta una preponderancia de las interrelaciones tipo combinadas, con un 33,8\% del total, a la que sigue la interrelación de tipo indirecta con las metas de los ODS, con un 28,8\%. El pilar de medio ambiente, al igual que el pilar social, manifiesta una preponderancia de las interrelaciones de tipo combinadas, con un $46,7 \%$. En este pilar también se encuentra una presencia significativa de interrelaciones indirectas, con un 23,3\%. En los pilares social y medioambiental, la ausencia de interrelación llega al 10\%, el doble que el pilar económico, cuyas ausencias representan el $5 \%$.

Dentro del pilar económico, los ODS 9, 10 y 12 cuentan con una amplia preponderancia de interrelaciones directas; incluso si se consideran solo estos tres Objetivos, las directas alcanzan un 76,7\%. Por su parte, el ODS 8 manifiesta un $60 \%$ de interrelaciones del tipo combinadas.

En el pilar social hay una amplia presencia de interrelaciones indirectas, especialmente en los ODS 3, 4 y 5, que, si se miran en conjunto, manifiestan un $60 \%$ de 
interrelaciones indirectas entre los ODS. El ODS 11 es el pilar social que cuenta con una mayor interrelación directa, con un $80 \%$ de interrelaciones de este tipo. En el caso del pilar social, causa alarma la situación del ODS 13 respecto al ODS 5 de igualdad de género, que cuenta con solo $50 \%$ de interrelaciones indirectas o ausentes. En el caso del ODS 1 tampoco se encuentran interrelaciones directas, sin embargo, un $80 \%$ de las interrelaciones corresponde a las de tipo combinada.

Sobre el pilar de medio ambiente, pudiera causar extrañeza la cantidad de interrelaciones directas, puesto que son planes y políticas correspondientes al mismo pilar. Sin embargo, se encuentra una alta interrelación de tipo combinada, donde las únicas ausencias se pueden ver en la Política Nacional para la Gestión de Riesgo de Desastres (PNGRD) que, debido a su naturaleza, no presenta metas y resultados concretos, pues estos están contenidos en su plan estratégico (PENGRD), en el que existe una interrelación de tipo combinada con los ODS del pilar medio ambiente.

Respecto al ODS 17, existe un amplio nivel de interrelaciones directas (90\%) respecto a los planes y políticas del ODS 13. Tan solo uno de los planes analizados no presenta acciones relacionadas directamente al cumplimiento con las metas del ODS 17.

Los dos planes con mayor interrelación directa con los ODS son el Plan de Acción de los Servicios de Infraestructura al Cambio Climático y el Plan de Acción Nacional de Cambio Climático, los cuales tienen interrelación directa con 8 de 16 ODS. En el caso de la política con mayor ausencia de interrelación, la PNGRD, como se mencionaba anteriormente, no se puede interpretar la interrelación de esta política por sí sola, puesto que las acciones específicas que busca esta política se encuentran plasmadas en el PENGRD. Este plan manifiesta la mayor interrelación de tipo combinada entre los ODS, con una interrelación de 11 de 16 ODS. Respecto a las interrelaciones indirectas, el Plan de Adaptación Sectorial Salud es el que tiene mayor interrelación de este tipo, con 7 de 11 ODS.

Los desafíos planteados por Chile en su informe nacional voluntario respecto al ODS 13 corresponden, en su mayoría, a desafíos que favorecerán la interrelación con los ODS del pilar económico — el cual es el más fuerte en interrelación directa-y, en segundo lugar, al pilar de medio ambiente. Si bien el país manifiesta desafíos que se interrelacionan directamente con el pilar social, como el fortalecimiento institucional sobre el cambio climático (directamente interrelacionado con el ODS 16), no hay una agenda nacional de cambio climático que se relacione directamente y en profundidad con los ODS del pilar social. 


\section{Análisis de Uruguay}

El análisis de planes en el caso de Uruguay contempló seis de las siete políticas públicas declaradas como logro en el Informe nacional voluntario Uruguay 2019,7 de las cuales, una es una política orientada al ODS 13, la Política Nacional de Cambio Climático y las cinco restantes corresponden a políticas y planes sectoriales. A partir del análisis se elaboraron una matriz resumen (figura 6) y los gráficos de la figura 7.

Figura 6. Uruguay, matriz de resultados

\begin{tabular}{|l|l|l|l|l|l|l|l|l|l|l|l|l|l|l|l|l|}
\hline Política o Plan/ODS & 1 & 2 & 3 & 4 & 5 & 6 & 7 & 8 & 9 & 10 & 11 & 12 & 13 & 14 & 15 & 16 \\
\hline Política Energética Nacional & & & & & & & & & & & & & & & & \\
\hline Política Nacional de Aguas & & & & & & & & & & & & & & & & \\
\hline $\begin{array}{l}\text { Plan Nacional Ambiental para } \\
\text { el Desarrollo Sostenible }\end{array}$ \\
\hline $\begin{array}{l}\text { Política Nacional de Gestión de Riesgos } \\
\text { de Desastres }\end{array}$
\end{tabular}

Fuente: Elaboración propia.

Los gráficos permiten apreciar similitudes entre los distintos pilares respecto a la presencia de interrelación directa, que es levemente mayor en los pilares económico y ambiental, con un 37,5\% y 38,9\% respectivamente. Sin embargo, el pilar económico se destaca además por la alta presencia de formas de interrelación combinada, que agrupa formas directas e indirectas de asociación, ya que sumadas a las directas caracterizan a un 70,8\% de las interrelaciones con ODS tipificados como económicos. Teniendo presente las relaciones indirectas, es posible señalar que un 95,8\% de las interrelaciones generan un fortalecimiento del pilar económico.

$7 \quad$ El plan Lineamientos Estratégicos hacia un Uruguay Agrointeligente no fue tomado en cuenta para este análisis, debido a la temporalidad y a su formato, que expresa logros y no metas. Este plan fue creado previamente a la elaboración de la Agenda 2030 y está más bien alineado con los Objetivos de Milenio. A diferencia de la Política Energética Nacional y la Política Nacional de Aguas, que tienen un horizonte temporal mayor, el plan de Lineamientos Estratégicos presenta un documento al año 2017 declarando los logros y experiencias desarrollados a partir del plan. 
Figura 7. Uruguay, análisis de pilares de desarrollo sostenible

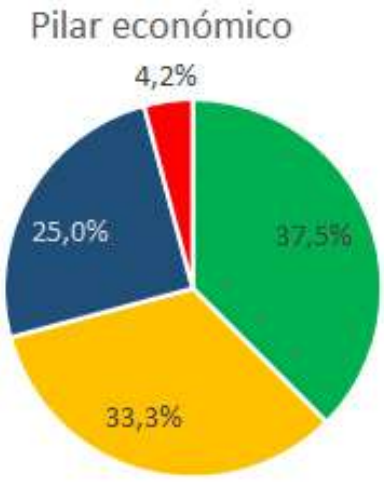

Pilar social

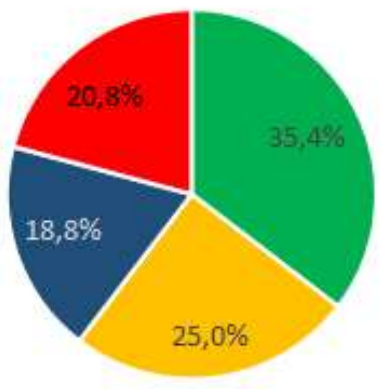

Pilar medio ambiente

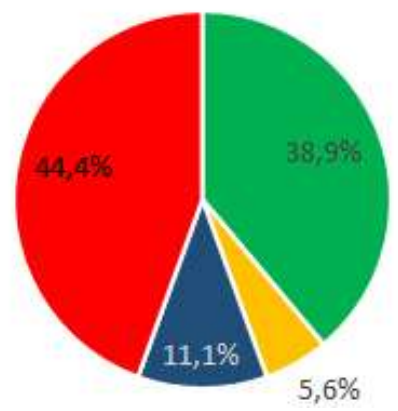

Referencias

- Interrelación directa con las metas delODS

= Interrelación tipo combinada (directa e indirecta con las metas del ODS)

- Interrelación indirecta con las metas del ODS

- Ausencia de interrelación

Fuente: Elaboración propia.

En el caso del pilar social, también priman las relaciones de tipo directo con un $35,4 \%$, que en conjunto con las relaciones combinadas alcanzan a un $60 \%$. Por otro lado, se destaca el respaldo de las relaciones indirectas, que corresponden a un 18,8\%.

Respecto del pilar de medio ambiente, es relevante la alta ausencia (44\% del total) de interrelaciones entre los planes y políticas vinculados al ODS 13 con los objetivos de esta materia. Esto es llamativo, considerando que el ODS 13 es un objetivo ambiental, por lo que podría asumirse que debiera tener una mayor relación con objetivos de esta categoría. Además, se destaca la presencia de interrelaciones directas con un 38,9\%, dando a entender que, si bien las medidas vinculadas a objetivos ambientales son escasas, cuando se presentan, suelen estar relacionadas directamente a metas específicas de estos objetivos. Este pilar es el que contempla una menor presencia de interrelaciones combinadas e indirectas, con cerca de un 6\% y $11 \%$ respectivamente.

Dentro del pilar económico es acentuada la interrelación con el ODS 9; la mitad de tipo directo y alcanza un $83 \%$ considerando relaciones combinadas, al igual que el ODS 12 , pero en este caso priman las relaciones combinadas. Por su parte, las relaciones del ODS 10 son principalmente indirectas. En el caso del ODS 8 las interrelaciones se dividen de forma equitativa entre las de tipo directo, combinado e indirecto. Dentro de este grupo, solo el ODS 12 no presenta relaciones en uno de sus planes.

Del conjunto de Objetivos que forman parte del pilar social, hay una alta proporción de relaciones directas en los ODS 7 y 16, con un $67 \%$ y $87 \%$, respectivamente. El 
ODS 4 presenta $50 \%$ de interrelaciones directas e indirectas, y es uno de los objetivos con mayor presencia de este tipo de vínculos. Los ODS 1 y 11 presentan una relación bastante heterogénea entre los distintos tipos de relaciones, pero con mayor presencia en las directas y las combinadas, con un 33\% en cada una. En el caso de los ODS 2 y 5 predomina la ausencia de interrelación, con un 50\%; en ambos casos, un $33 \%$ de relaciones indirectas se insertan en el ámbito de acción de estos objetivos.

Analizando en detalle el pilar ambiental, se constata en este la predominancia de ausencia de relaciones con estos objetivos. Esto se aprecia en los casos de los ODS 14 y 15 , con un $67 \%$ y $50 \%$, respectivamente. Para el ODS 6 hay mayor presencia de interrelaciones directas (50\%), destacando la Política Nacional de Aguas con una fuerte orientación a este objetivo.

El ODS 17 se compone tanto de relaciones directas como de relaciones combinadas, en ambos casos con un 50\%. Esto muestra una presencia de relaciones directas en todos los planes analizados.

Respecto a las políticas declaradas como logros, se destaca el Plan Nacional Ambiental para el Desarrollo Sostenible, con un 50\% de relaciones directas y un 38\% combinadas, que presenta con todos los objetivos algún tipo de relación, ya sea directa, combinada o indirecta.

\section{Conclusiones}

En relación con la pregunta central del presente estudio, sobre cuán interconectadas se encuentran las acciones de los gobiernos con las áreas de desarrollo propuestas por los ODS, con base en el ODS13, se concluye, de manera comparativa:

Los actuales planes y políticas de Chile y de Uruguay vinculados al ODS 13 presentan interrelaciones con los demás objetivos de la Agenda 2030; de forma conjunta, e independiente del tipo de interrelaciones, alcanzan un $81 \%$ en el caso de Uruguay y un 91\% en el caso de Chile. En ambos casos el énfasis está puesto en el pilar económico, Esta tendencia se acentúa en la agenda de Chile, en donde las relaciones directas y combinadas toman gran relevancia.

Particularmente, en el pilar social predomina la ausencia de relaciones para el caso de Uruguay; sin embargo, este país presenta principalmente relaciones directas, es decir, vinculadas a metas específicas, en comparación con Chile. Por esto, el pilar social es un aspecto que se debe fortalecer en los planes que se consideren como logro del ODS 13 vinculado a la acción climática. Esto cobra especial importancia, teniendo en cuenta la necesidad de erradicar injusticias sociales relativas a los efectos asociados al cambio climático y las complicaciones que conlleva este fenómeno respecto al logro de objetivos como, por ejemplo, los obstáculos que impone a la reducción de la pobreza (ODS 1) y los 
perjuicios que genera a la seguridad alimentaria (ODS 2) (Intergovernmental Panel on Climate Change [IPCC], 2014).

En ambos países, uno de los objetivos que requiere más atención dentro de este pilar corresponde al ODS 5, vinculado a la equidad de género. En el caso de Chile, resulta más preocupante, ya que no cuenta con interrelaciones combinadas ni directas. Como señala ONU Mujeres respecto al ODS 13, las mujeres están vinculadas al ámbito de la agricultura (interrelación directa con el ODS 2), forrajeo (interrelación directa con los ODS 7 y 15), recolección de agua (interrelación directa con el ODS 6) y la pesca a pequeña escala (interrelación directa con el ODS 14) (ONU Mujeres, 2018, p. 119). Todos estos ámbitos son cubiertos en los planes sectoriales y en los planes de acción y adaptación del cambio climático; sin embargo, no se consideran acciones de género en ningún objetivo o meta vinculados a estos.

Por otra parte, en la PNGRD y su PENGRD, también se presenta el espacio y desafío de incluir metas específicas relacionadas con acciones de género, tomando en cuenta los ámbitos mencionados anteriormente. Asimismo, es necesario adoptar acciones de género en la agenda de gestión de riesgo de desastres, puesto que, por ejemplo, es 14 veces más probable que mujeres y niños fallezcan durante un desastre que los hombres, lo que requiere una acción pública urgente con miras a reducir la desigual vulnerabilidad según el género frente a los desastres (ONU Mujeres, 2018).

En el caso de Uruguay si bien se presenta la Estrategia Nacional para la Igualdad de Género, la cual tiene una alta interrelación agregada con el resto de los Objetivos, da cuenta de un manejo focalizado en una temática que es transversal en las sociedades. Esto resulta preocupante, en la medida que las mujeres son un grupo de la población identificado como más vulnerable a los efectos del cambio climático (United Nations, 2020), por lo que se requiere su consideración en las diferentes políticas que se impulsen vinculadas al tema.

A partir de los análisis de interrelación de planes y políticas del ODS 13 respecto a los demás ODS es posible dilucidar que la agenda chilena de acción climática está fuertemente ligada a lo económico, dejando de lado los componentes sociales. Este esquema no es sostenible si se vuelve a lo planteado por el Centro de Resiliencia de Estocolmo, sobre que lo económico está inserto en lo social y, para que la biosfera prospere, debe haber un equilibrio entre estos componentes. Sin embargo, debido al sistema económico-político neoliberal ${ }^{8}$ que impera en Chile, no es una sorpresa la preponderancia de sistema neoliberal donde además el Estado adquiere un rol subsidiario. Saavedra (2014) señala que "el modelo económico-político chileno es el de una economía de mercado que protege fuertemente la libertad de emprendimiento y los derechos de propiedad, asignando al Estado un rol subsidiario y no empresarial. El Estado en Chile tiene un papel regulador en tanto corrector de fallas de mercado y fallas 
lo económico por sobre lo social, que se manifiesta incluso en planes y políticas de cambio climático. El Estado chileno tiene un rol subsidiario, el cual se debe superar con miras a fortalecer la cohesión social y acceso al bienestar, para otorgar a la población un acceso universal a los sistemas de protección social, bienes sociales y públicos esenciales (Comisión Económica para América Latina y el Caribe [CEPAL], 2019). De esta manera, la agenda climática se vería fortalecida en relación con lo social, sin dejar de lado los grandes avances que ha hecho el país respecto al ámbito de interrelación económica con los planes y políticas del ODS 13.

Por último, el pilar ambiental se presenta en Chile con mayor fuerza en lo que respecta al conjunto de relaciones directas y combinadas, que alcanzan un $66 \%$. En cambio, en Uruguay se hace notar la ausencia de relaciones, a pesar de que presenta un nivel de interrelaciones directas mayor que Chile. Esto refleja una mayor intención de establecer medidas ambientales vinculadas a las metas de la Agenda 2030, a pesar de que es necesaria su amplificación a los distintos sectores, de modo que la temática ambiental sea un eje transversal en las políticas de este país. Esta debilidad es relevante, considerando que las políticas y planes de estos países son presentados en el marco del objetivo de acción climática (ODS 13), que se constituye dentro de este pilar pero que no dialoga con los otros objetivos de este. Esta materia suele tener menor cabida en la agenda pública de los países y de la actividad humana en general, y está vinculada a las causas de una de las mayores crisis que amenaza la sostenibilidad de los sistemas ambientales y sociales.

Dada la alta presencia de interrelaciones directas y combinadas del ODS 13 con otros objetivos, el presente estudio releva la importancia de impulsar planes y políticas vinculadas a la acción climática en Chile y en Uruguay. Las interrelaciones deben estar orientadas desde una mirada integral de la Agenda 2030, para permitir el fortalecimiento de los demás objetivos. Sin embargo, esta integración se debe dar de forma intencionada, ya que puede no ser equitativa entre los distintos pilares, como en los casos estudiados, en que a través de estas medidas se hace más robusto el pilar económico, en comparación con los pilares social y ambiental. Si se retoman las orientaciones del Centro de Resiliencia de Estocolmo sobre el establecimiento de un pilar con base en la biosfera, luego el pilar social y por último el pilar económico, es indispensable señalar la necesidad de planificar e implementar la política pública resguardando dicho orden de prioridades. Es importante avanzar en conjunto, «sin que nada ni nadie se quede atrás» para alcanzar un verdadero desarrollo sostenible.

sociales, es un sistema económico-político es de tipo 'liberal', a diferencia de otros modelos capitalistas 'coordinados' y 'jerárquicos'”' (p. 118). 


\section{Referencias bibliográficas}

Banco Mundial. (2013). New country classifications. Recuperado de https://blogs.worldbank.org/opendata/new-country-classifications.

Comisión Económica para América Latina y el Caribe. (2019). Panorama social de América latina. Santiago: Cepal.

Comisión Mundial sobre el Medio Ambiente y el Desarrollo. (1987). Nuestro futuro común: informe de la Comisión Mundial sobre el medio ambiente y el desarrollo. Recuperado de https://cpr.org.ar/wp-content/uploads/media/uploads/documents/ investigacionpidc/nacionesunidas-informe-de-la-comision-mundial-sobre-elmedio- ambiente-y-el-desarrollo.pdf.

Duarte, C. (2006). Cambio global: Impacto de la actividad humana sobre el sistema Tierra. Madrid: Consejo Superior de Investigaciones Científicas.

Gobierno de Chile. (2019). $2^{\circ}$ Informe Nacional Voluntario: Chile 2019. Recuperado de https://sustainabledevelopment.un.org/content/documents/23507Informe_Nacional_Voluntario_CHILE_Junio_2019_final_1.pdf.

Intergovernmental Panel on Climate Change. (2014). Cambio climático 2014: Informe de síntesis. Recuperado de https://www.ipcc.ch/site/assets/uploads/2018/02/ SYR_AR5_FINAL_full_es.pdf.

Lobos Alva, I., Martin, P., y Purkey, D. (2019). Análisis de interacciones de los ODS. Colombia: Stockholm Environment Institute.

Lur Consultores. (2019). Desarrollo Sostenible: los 17 ODS y la biosfera. Recuperado de https://www.lurconsultores.com/gestion-ambiental/desarrollo-sostenible/desarrollo-sostenible-ods-biosfera\#.XymHXShKg2w.

ONU Mujeres (2018). Hacer las promesas realidad: La igualdad de género en la agenda 2030 para el desarrollo sostenible. Recuperado de http://www.unwomen.org/media/headquarters/attachments/sections/library/publications/2018/sdg-report-gender-equality-in-the-2030-agenda-for-sustainable-development-2018es.pdf.

Presidencia República Oriental del Uruguay. (2019). Objetivos de desarrollo sostenible: Informe Nacional Voluntario Uruguay 2019. Recuperado de https://sustainabledevelopment.un.org/content/documents/23786Informe_Nacional_Voluntario_Uruguay_2019.pdf.

Programa de las Naciones Unidas para el Desarrollo. (2020). Objetivo 13: Acción por el clima. Recuperado de https://www.undp.org/content/undp/es/home/sustainable-development-goals/goal-13-climate-action.html.

Programa de las Naciones Unidas para el Desarrollo. (2017). Bienestar más allá del ingreso. Nueva York: PNUD. 
Rockström, J., y Sukhdev, P. (2016). How food connects all the SDGs: Stockholm resilience centre. Recuperado de https://www.stockholmresilience.org/research/researchnews/2016-06-14-how-food-connects-all-the-sdgs.html.

Saavedra, E. (2014). El modelo económico-político de Chile: Desarrollo institucional en la encrucijada. Economía y Política, 1(1), 115-146.

Stockholm Resilience Centre. (2016). La Agenda 2030 y los ecosistemas Un documento para la discusión de los vínculos entre las Metas de Aichi para la Diversidad Biológica y los Objetivos de Desarrollo Sostenible. Recuperado de https://swed.bio/wp-content/uploads/2018/06/SUNI-199-Rapport-2030-Agenda-and-Ecosystems_ESP.pdf.

United Nations. (2020). Gender, climate \& security: Sustaining inclusive peace on the frontlines of climate change. Recuperado de https://www.unwomen.org/-/media/headquarters/attachments/sections/library/publications/2020/gender-climate-and-security-en.pdf?la=en\&vs=215.

Weitz, N., Carlsen, H., Nilsson, M., y Skånberg, K. (2018). Towards systemic and contextual priority setting for implementing the 2030 Agenda. Sustainability Science, 13, 531-548. 